\title{
RESPONSE OF GROWTH, YIELD AND FRUIT QUALITY OF "ANNA" APPLE TREES TO FOLIAR SPRAY WITH SOME PLANT GROWTH PROMOTING RHIZOBACTERIA AS A SUBSTITUTE TO SYNTHETIC BIOSTIMULANTS \\ M. Nour El-Din* ; M. A. Gabr** and M.Y. Abou-zeid* \\ * Soil, Water and Environment Research Institute- Giza -Egypt \\ ${ }^{* *}$ Horticulture Research Institute - Giza - Egypt
}

\begin{abstract}
Many synthetic biostimulants containing amino acids, macro- and microelements, humic acids and, sometimes, algae extracts have been used effectively for stimulating and increasing of fruit trees yield. The foliar spray with some PGPR (plant growth promoting rhizobacteria) has proved high potentiality in stimulating and increasing plant yield. The current study is concerned with the use of some PGPR types (Azospirillum brasilense, Pseudomonas fluorescens and Bacillis polymyxa) as an alternative to some commercial synthetic biostimulants i. e. Jisamar (J) and Furdos $(F)$ in stimulating and improving yield of "Anna" apple trees.

The study field of apple trees (eight years old) was chosen at El-Bostan area, El-Behira Governorate, Egypt. The trees were sprayed twice with the different stimulators, the first was at fruit set and the second was done after 30 days from fruit set. The control trees were sprayed with water.

The microorganisms which were used for spray were counted in the plant phyllosphere and they showed heavy increase in the phyllosphere of the treated plants. The spray with the synthetic biostimulants showed remarkable improvement to the plant morphological characteristics, e. g. shoot length, shoot diameter, leaf area, leaf dry weight and leaf content of chlorophyll a, b and total chlorophyll. While, specific leaf weight (SLW) did not significantly change. Likewise, the spray with bacterial biostimulants proved similar potentiality as synthetic ones, and even, sometimes, surpassed them. The spray with Azospirillum brasilense gave the highest fruit yield; 45.1 and $41.2 \mathrm{~kg} /$ tree versus 33.5 and $32.0 \mathrm{~kg} /$ tree for control treatment in 2009 and 2010 seasons, respectively. The spray with Jisamar and Furdos gave 37.4 and 33.2 $\mathrm{kg} /$ tree and 36.5 and $34.7 \mathrm{~kg} /$ tree, respectively. The experimental treatments, raised the net return (£E/feddan) over that of control. Azospirillum brasilense spray attained the highest net return i. e. 21595 versus 16660, 16870 and $15650 £$ £E/feddan for treatments of Jisamar, Fordose and control, respectively. Therefore, the substitution of synthetic biostimulant products with biological ones may be suitable for improving the plant growth, increasing productivity and raising the net return per feddan of Anna apple trees.
\end{abstract}

\section{INTRODUCTION}

The overwhelming increase of population and exacerbate of poverty and hungry problems in the world needs hard and continuous work to increase agricultural productivity. For a lot of reasons, horizontal expansion of agriculture represent a difficult equation for many countries of the world, therefore the vertical increase in plant productivity is the suitable solution.

Despite the balanced plant nutrition is of great importance for plant productivity, the application of some synthetic stimulants had the positive and 


\section{Nour El-Din et al.}

effective influence in increasing plant growth and productivity. Many of these stimulants contain amino acids, vitamins, humic acids, sea weeds extracts, plant phytohormones and sometimes microelements (Ferrini and Nicese, 2002). Spinelli et al. (2010) treated the strawberry with "Actiwave" which is a product derived from the algae Ascophyllum nodosum. Spray with "Actiwave" enhanced the tree growth and yield and had a significant effect on reducing the negative effect of alternative bearing. They claimed that the biostimulant Actiwave may represent a promising strategy to reduce the use of phytochemicals in agriculture. They indicated that Actiwave application as a spray to the strawberry increased the mineral nutrient uptake, chlorophyll content and the abiotic stress tolerance.

Other authors found significant increase in plant growth and productivity due to spray with different types of PGPR cultures. Karakurt and Aslantas (2010) demonstrated that the spray of apple tree with some PGPR (Bacillus subtilis OUS-142 and Pseudomonas putida BA-8) enhanced tree growth and nutrient uptake. Erturk et al. (2011) observed that spray of hazelnut seedlings with $B$. lentus, $B$. atrophaeus and other types of PGPR significantly increased the plant growth parameters. The foliar spray of apple trees with three Bacillus sp. was also found by Ryu et al. (2011) to increase tree growth, fruit yield and quality.

The PGPR had multi-mechanisms for enhancing plant growth such as fixing atmospheric nitrogen (Saharan and Nehra, 2011); facilitate nutrient uptake (Martinez-Viveros et al., 2010); solubilizing phosphates (Oliveira et al., 2009); producing plant phytohormones (Spaepen et al., 2008); regulate plant ethylene level (Govidasamy et al., 2008); resist plant pathogens (Van Loon, 2007) and produce antibiotics which reflected on plant growth, yield and quality (Esitken, 2011).

Therefore, the present investigation aimed to study the significance of spray of apple trees with some PGPR bacterial biostimulants as an alternative to synthetic biostimulants i. e. Jisamar and Furdose.

\section{MATERIALS AND METHODS}

\section{Materials:}

Microbial media:

1- Azospirillum: Semi solid Döbereiner medium (Döbereiner et al., 1976) contained (g/liter of distilled water): Malic acid, 5.0; $\mathrm{KH}_{2} \mathrm{PO}_{4}, 0.4$; $\mathrm{K}_{2} \mathrm{HPO}_{4}, \quad 0.1 ; \mathrm{MgSO}_{4} .7 \mathrm{H}_{2} \mathrm{O}, 0.2 ; \mathrm{NaCl}, 0.1 ; \quad \mathrm{CaCl}_{2} .7 \mathrm{H}_{2} \mathrm{O}, \quad 0.02 ;$ $\mathrm{FeCl}_{3} .6 \mathrm{H}_{2} \mathrm{O}, 0.01 ; \mathrm{Na}_{2} \mathrm{MoO}_{4} .2 \mathrm{H}_{2} \mathrm{O}, 0.002 ;$ Agar, 1.75 .

2- Bacillus: N-deficient medium for Bacilli (Hino and Wilson, 1958) was composed of solution A: It contains ( $\mathrm{g} / 500 \mathrm{ml}$ distilled water): $\mathrm{MgSO}_{4}$. $7 \mathrm{H}_{2} \mathrm{O}, 0.5$; Sucrose, $20.0 ; \mathrm{NaCl}, 0.01 ; \mathrm{FeSO}_{4}, 0.02 ; \mathrm{Na}_{2} \mathrm{MoO}_{4} .2 \mathrm{H}_{2} \mathrm{O}$, $0.005 ; \mathrm{CaCO}_{3}, 0.2$ and solution $\mathrm{B}$ which contains Para amino benzoic acid, $10 \mu \mathrm{g}$; Biotin, $5 \mu \mathrm{g}$; $\mathrm{K}_{2} \mathrm{HPO}_{4}, 6.81 \mathrm{~g} ; \mathrm{KH}_{2} \mathrm{PO}_{4}, 1.43 \mathrm{~g}$; distilled water $500 \mathrm{ml}$. The two solutions were mixed after autoclaving at $1.5 \mathrm{~atm}$. for 15 minutes. 
3- Pseudomonas: King $\square s$ medium (King et al., 1954) was composed of (g/liter distilled water): sucrose, $20.0 \mathrm{~g}$; peptone, $5.0 \mathrm{~g} ; \mathrm{K}_{2} \mathrm{HPO}_{4}, 0.5 \mathrm{~g}$; Agar , $20.0 \mathrm{~g}$; distilled water $1000 \mathrm{ml}, \mathrm{pH}, 7.2(0.1 \mathrm{~N} \mathrm{KOH})$.

Jisamar: is a commercial synthetic biostimulant contains seaweed extract $(20.5 \%)$, free amino acids (6.5\%), total nitrogen (5.8\%), phosphorus $(3 \%)$, boron $(0.17 \%)$ and potassium $(4.6 \%)$.

Furdose: : is a commercial synthetic biostimulant contains humic and vulvic acids $(22 \%)$, natural and organic substances $(40 \%)$, free amino acids (14.6\%), N, (4.5\%), P (3.8), K (5\%), Ca (0.4\%), Mg (0.4\%), Fe (0.1\%), Mn (15 ppm), Zn (20 ppm), Cu (15 ppm).

This study has been carried out on eight years old "Anna" apple trees budded on Malus rootstock during 2009 and 2010. Trees were grown at Elbostan region of Elbehira Governorate. Drip fertigation system was applied for irrigation and fertilization. The experimental soil analysis is shown below in Table 1.

Table 1: Some chemical and physical analysis characteristics of the experimental soil

\begin{tabular}{|c|c|c|c|c|c|c|c|c|c|}
\hline Sand \% & \multicolumn{2}{|c|}{ Silt \% } & Clay \% & \multicolumn{2}{|c|}{ Texture } & O.M \% & $\mathrm{pH}$ & \multicolumn{2}{|c|}{$E C\left(d s \cdot m^{-1}\right)$} \\
\hline 80.2 & 8 & & 11.3 & Sandy $\mathrm{C}$ & y loam & 0.63 & 8.1 & & .17 \\
\hline \multicolumn{3}{|c|}{ Cations (mg./L) } & \multicolumn{4}{|c|}{ Anions (mg./L) } & \multicolumn{3}{|c|}{ Macro-nutrient (ppm) } \\
\hline $\mathrm{Na}^{+}$ & $\mathrm{Ca}^{++}$ & $\mathrm{Mg}^{++}$ & $\mathrm{CO}_{3}{ }^{--}$ & $\mathrm{HCO}_{3}^{-}$ & $\mathrm{Cl}^{-}$ & $\mathrm{SO}_{4}^{--}$ & $\mathbf{N}$ & $\mathbf{P}$ & $\mathrm{K}$ \\
\hline 5.89 & 10.42 & 3.91 & - & 1.37 & 12.41 & 6.72 & 139 & 7 & 86 \\
\hline
\end{tabular}

\section{Methods:}

Treatments were arranged in a random order on the selected trees. Single tree plot with 3 replicates for each treatment was arranged in random complete blocks design.

All trees were subjected with common regional horticultural practices, while treatments were applied as follows:

(A): Trees were sprayed with a suspension of Azospirillum brasilense.

(P): Trees were sprayed with a suspension of Pseudomonas fluorescens.

(B): Trees were sprayed with a suspension of Bacillus polymyxa

(J): Trees were sprayed with a suspension of Jisamar.

(F): Trees were sprayed with a suspension of Furdose.

$(A+J)$ : Trees were sprayed with a suspension of Azospirillum brasilense and Jisamar.

$(\mathrm{A}+\mathrm{F})$ : Trees were sprayed with a suspension of Azospirillum brasilense and Furdose.

$(\mathrm{P}+\mathrm{J})$ : Trees were sprayed with a suspension of Pseudomonas fluorescens and Jisamar

$(\mathrm{P}+\mathrm{F})$ : Trees were sprayed with a suspension of Pseudomonas fluorescens and Furdose.

$(B+J)$ : Trees were sprayed with a suspension of Bacillus polymyxa and Jisamar. 


\section{Nour El-Din et al.}

$(\mathrm{B}+\mathrm{F})$ : Trees were sprayed with a suspension of Bacillus polymyxa and Furdose.

(M): Trees were sprayed with a suspension of the bacterial mixture.

$(\mathrm{M}+\mathrm{J})$ : Trees were sprayed with a suspension of the bacterial mixture and Jisamar.

$(\mathrm{M}+\mathrm{F})$ : Trees were sprayed with a suspension of the bacterial mixture and Furdose.

(C): Control trees sprayed with tap water

The Microbial inoculants were prepared and provided by Biofertilizers Production Unit, Soil, Water and Environment Research Institute, ARC, Egypt. Bacterial suspensions were diluted by mixing of $400 \mathrm{ml}$ of bacterial stocks with $9 \mathrm{~L}$ of water per 3 trees of each treatment. Each of the used bacterial species was grown on its specific medium. Bacillus polymyxa was grown up on liquid medium of Hino and Wilson (1958) for 3 days at $30^{\circ} \mathrm{C}$. Azospirillum brasilense was grown up for 3 days at $30^{\circ} \mathrm{C}$ on semi solid Döbereiner medium (Döbereiner et al., 1976) and Pseudomonas fluorescense was grown up for 3 days at $30^{\circ} \mathrm{C}$ on King s medium (King et al., 1954). Each tree was sprayed twice, at fruit sitting and 30 days later for both seasons.

Viable counts of $P$. fluorescense, $A$. brasilense and $B$. polymyxa:

$P$. fluorescense, $A$. brasilense and $B$. polymyxa viable count in the phylosphere of apple tree were determined, 30 days before fruit maturity at the second season, using respective media.

Estimation of plant growth promoting substances by bacteria:

Bacterial strains were tested for their capability to produce indole acetic acid (IAA) (Bric et al., 1991), were grown on Tryptone Soya Agar Medium (TSA) (Difco, 1984). Quantitative determination of IAA was performed according to Glickmann and Dessoux (1995). Total gibberellins were measured by the procedure of Udagwa and Kinoshita (1961).

Three branches, five years old, in different directions on each tree were selected and labeled to estimate growth parameters. All current shoots developed on these branches were measured to get shoot length $(\mathrm{cm})$. $\mathrm{Li}$ Core-3100 Areameter was used to measure detached leaves of nine shoots (three shoots per branch) to get area per leaf $\left(\mathrm{cm}^{2}\right)$. Leaves were dried at $70^{\circ} \mathrm{C}$ and weighted to get dry weight (mg.) and then specific leaf weight (SLW) was calculated as $\left(\mathrm{mg} / \mathrm{cm}^{2}\right)$ according to Ferree and Forshey (1988).

Spectrophotometer was used to estimate chlorophyll a and chlorophyll b which extracted from fresh leaves with di-methyl formamide (DMF) as described by Rami and Porath (1980). The concentration of chlorophyll a and chlorophyll b and its total value were calculated by Rami's formulas as $(\mu \mathrm{g} /$ $\mathrm{ml}$ ) (Rami 1982) while results were presented as $\left(\mathrm{mg} / \mathrm{cm}^{2}\right)$.

Fruits were picked at maturity stage and weighted and counted. Fruit pulp texture (firmness) was recorded by using Lfra texture analyzer instrument. The results were expressed as a resistance force of the fruit to the penetrating tester $\left(\mathrm{g} / \mathrm{cm}^{2}\right)$ according to Harold (1985). Fruit skin color measurements $\left(a^{*}, b^{*}, L^{*} \& H^{\circ}\right)$ were determined using Minolta colorimeter (Minolta Co. Ltd., Japan). The instrument estimated skin color of fruits with 
color metric CIE Lab method where $L^{*}$ measure lightness scale readings and the two coordinates $a^{*}$ and $b^{*}$ included. Positive values of $a^{*}$ is a measure of redness and becomes greenish measure when values changed into negative, while $b^{*}$ of yellowness and blueness $\left(-b^{*}\right)$ on the Hue circle. The Hue angle $\left[\mathrm{H}^{\circ}=\operatorname{arc} \tan \left(\mathrm{b}^{*} / \mathrm{a}^{*}\right)\right]$ describe the relative amounts of redness and yellowness where point at $0^{\circ} / 360^{\circ}$ is defined for red $/ \mathrm{magenta}, 90^{\circ}$ yellow, $180^{\circ}$ for green and $270^{\circ}$ for blue color (McGuire, 1992 and Voss, 1992). Soluble solids content (SSC) was determined by using a hand refractometer and total acidity percentage was estimated in filtered juice according to A.O.A.C. (1990).

Statistical analysis:

Data obtained were subjected to the analysis of variance and treatment means were compared using the L.S.D methods according to Steel and Torrie (1980).

\section{RESULTS AND DISCUSSION}

The synthetic and/or bacterial biostimulants foliar spray treatments had an effective role in increasing shoot length of apple trees (Table 2). At the first season, Jisamar treatment attained the highest and significant increase $(64.83 \mathrm{~cm})$ followed by the treatment of $P$. fluorescens which gave, a significant increase of $64.63 \mathrm{~cm}$ compared to water spray (control) treatment $(56.36 \mathrm{~cm})$. The second season trend was similar to that of the first one. The studied treatments exhibited not significant increments in shoot diameter. The spray with Furdose, Jisamar and bacterial inoculants and their mixtures with PGPR biostimulants had a great effect in increasing leaf area and dry weight. In most cases, the treatments achieved consistent, remarkable and significant increases over control. Specific leaf weight (SLW) data did not show significant differences.

Spray of fruit trees with compounds containing amino acids, plant phytohormones, humate, $\mathrm{N}, \mathrm{P}, \mathrm{K}$ and some microelements contribute in improving tree growth, promote flowering process and fruit setting, which consequently, reflected positively on the fruit yield (Eissa, 2003). Likewise, foliar application with some PGPR types (Azotobacter chroococcum) gave results similar to the action of these treatments (Nour El-Din, 2006). The results of the present study showed that foliar spray with some synthetic plant stimulants (Furdose and Jisamar) which contain sea weeds extracts, amino acids, low percentages of $\mathrm{N}, \mathrm{P}$ and $\mathrm{K}$ with microelements had the positive effect on shoot length, stem diameter, leaf area and leaf dry weight. The spray with the varied PGPR types alone or mixed with the synthetic biostimulants, similarly, increased these characteristics of apple trees, in some cases surpassed synthetic ones. This promoting influence is attributed to the plant phytohormones, amino acids, vitamins and macro and microelements contained in bacterial biostimulants. Fathi et al. (2002) found that spray with $\mathrm{GA}_{3}$, K-humate and ascorbic acid significantly increased shoot length, leaf area of "Desert Red" peach and increased leaf area and leaf dry weight of "Anna" apple. Esitken et al. (2004), also, reported that spray with 
Bacillus OSU142 at full bloom, 30 and 60 days after blooming increased shoot length and growth of apricot tree especially when sprayed at full bloom.

Table 2: Effect of spray with some synthetic and some bacterial biostimulants (PGPR cultures) on "Anna" apple vegetative characteristics

\begin{tabular}{|c|c|c|c|c|c|c|c|c|c|c|}
\hline \multirow[t]{2}{*}{ Treatments } & \multicolumn{2}{|c|}{$\begin{array}{c}\text { Shoot } \\
\text { length }(\mathrm{cm})\end{array}$} & \multicolumn{2}{|c|}{$\begin{array}{l}\text { Shoot } \\
\text { Diameter } \\
(\mathbf{c m})\end{array}$} & \multicolumn{2}{|c|}{$\begin{array}{l}\text { Leaf dry } \\
\text { weight (g) }\end{array}$} & \multicolumn{2}{|c|}{$\begin{array}{l}\text { Leaf area } \\
\left(\mathrm{cm}^{2}\right)\end{array}$} & \multicolumn{2}{|c|}{$\begin{array}{c}(\mathrm{SLW}) \\
\left.(\mathrm{mg} \mathrm{cm})^{2}\right)\end{array}$} \\
\hline & 2009 & 2010 & 2009 & 2010 & 2009 & 2010 & 2009 & 2010 & 2009 & 2010 \\
\hline A & $\begin{array}{l}61.2 \\
a b c\end{array}$ & $\begin{array}{c}58.43 \\
a b c\end{array}$ & $\begin{array}{l}1.59 \\
a b\end{array}$ & $\begin{array}{l}1.53 \\
a b\end{array}$ & $\begin{array}{c}0.370 \\
\text { bcd }\end{array}$ & $\begin{array}{c}0.343 \\
a b c\end{array}$ & $\begin{array}{c}33.93 \\
a b c\end{array}$ & $\begin{array}{c}30.63 \\
\text { bcd }\end{array}$ & $\begin{array}{c}10.84 \\
\text { bcd }\end{array}$ & $\begin{array}{c}11.16 \\
b-e\end{array}$ \\
\hline$A+J$ & $\begin{array}{c}57.56 \\
b c\end{array}$ & $55.57 \mathrm{c}$ & $\begin{array}{c}1.62 \\
a b\end{array}$ & $\begin{array}{c}1.57 \\
a b\end{array}$ & $\begin{array}{c}0.363 c \\
d\end{array}$ & $\begin{array}{c}0.333 b \\
c\end{array}$ & \begin{tabular}{|l|}
34.8 \\
$a b c$
\end{tabular} & $\begin{array}{c}32.43 a \\
b c\end{array}$ & $10.38 d$ & $\begin{array}{c}10.37 d \\
-e\end{array}$ \\
\hline$A+F$ & $\begin{array}{c}60.16 \\
a b c\end{array}$ & $\begin{array}{c}57.17 b \\
c\end{array}$ & $\begin{array}{c}1.69 \\
a b\end{array}$ & $\begin{array}{c}1.57 \\
a b\end{array}$ & $\begin{array}{l}0.39 \\
a b c\end{array}$ & $\begin{array}{c}0.377 a \\
b\end{array}$ & $37.06 a$ & $\begin{array}{c}34.13 a \\
b\end{array}$ & $\begin{array}{c}10.66 \mathrm{c} \\
\mathrm{d}\end{array}$ & $\begin{array}{c}10.86 b \\
e\end{array}$ \\
\hline B & $\begin{array}{c}61.93 \\
a b\end{array}$ & $\begin{array}{c}53.73 a \\
b c\end{array}$ & $1.75 \mathrm{a}$ & $1.64 \mathrm{a}$ & $0.347 d$ & $\begin{array}{c}0.320 c \\
d\end{array}$ & $\begin{array}{c}32.23 a \\
-d\end{array}$ & $\begin{array}{c}28.73 c \\
-f \\
\end{array}$ & $\begin{array}{c}10.71 \mathrm{c} \\
\mathrm{d}\end{array}$ & $\begin{array}{c}11.11 \mathrm{~b} \\
-\mathrm{e}\end{array}$ \\
\hline $\mathbf{B}+\mathrm{J}$ & $56.1 \mathrm{c}$ & $\begin{array}{c}57.47 b \\
c\end{array}$ & $\begin{array}{c}1.63 \\
a b\end{array}$ & $\begin{array}{c}1.51 \\
a b\end{array}$ & $0.347 d$ & $\begin{array}{c}0.317 \mathrm{c} \\
\mathrm{d}\end{array}$ & $\begin{array}{c}31.46 a \\
-d\end{array}$ & \begin{tabular}{|c|}
$28.73 c$ \\
$-f$ \\
\end{tabular} & $\begin{array}{c}11.00 \mathrm{~b} \\
\mathrm{~cd}\end{array}$ & $10.09 \mathrm{e}$ \\
\hline$B+F$ & $\begin{array}{c}61.06 \\
a b c\end{array}$ & $55.43 c$ & $\begin{array}{c}1.69 \\
a b\end{array}$ & $\begin{array}{c}1.56 \\
a b\end{array}$ & $\begin{array}{l}0.39 \\
a b c\end{array}$ & $\begin{array}{c}0.373 a \\
b\end{array}$ & $\begin{array}{c}31.7 \mathrm{a}- \\
\mathrm{d}\end{array}$ & $\begin{array}{c}29.3 b- \\
e\end{array}$ & $\begin{array}{c}12.04 a \\
d\end{array}$ & $\begin{array}{c}12.88 a \\
b c\end{array}$ \\
\hline $\mathbf{P}$ & $\begin{array}{c}64.63 \\
a\end{array}$ & $\begin{array}{c}60.8 \\
a b\end{array}$ & $\begin{array}{c}1.66 \\
a b\end{array}$ & $\begin{array}{c}1.57 \\
a b\end{array}$ & $\begin{array}{c}0.397 a \\
b c\end{array}$ & $\begin{array}{c}0.370 a \\
b\end{array}$ & $35.96 a$ & $36.07 a$ & $\begin{array}{c}11.24 b \\
c d\end{array}$ & $\begin{array}{c}10.34 d \\
e\end{array}$ \\
\hline$P+J$ & $\begin{array}{l}60.2 \\
a b c \\
\end{array}$ & $\begin{array}{c}56.67 b \\
c\end{array}$ & \begin{tabular}{|c|}
1.73 \\
$a b$
\end{tabular} & $1.60 \mathrm{a}$ & $\begin{array}{c}0.370 \mathrm{~b} \\
\mathrm{~cd}\end{array}$ & $\begin{array}{c}0.350 a \\
b c\end{array}$ & $36.1 \mathrm{a}$ & $\begin{array}{c}33.10 \mathrm{a} \\
\mathrm{bc}\end{array}$ & $10.29 \mathrm{~d}$ & $\begin{array}{c}10.56 c \\
\text { de }\end{array}$ \\
\hline$P+F$ & $\begin{array}{l}61.6 \\
a b c\end{array}$ & $55.83 c$ & $\begin{array}{c}1.65 \\
a b\end{array}$ & $\begin{array}{c}1.52 \\
a b\end{array}$ & $\begin{array}{c}0.410 a \\
b\end{array}$ & $0.387 a$ & $\begin{array}{c}35.26 a \\
b\end{array}$ & $35.57 a$ & $\begin{array}{c}11.70 b \\
c d\end{array}$ & $\begin{array}{c}10.91 b \\
-e\end{array}$ \\
\hline$M$ & $\begin{array}{c}58.53 \\
\text { bc } \\
\end{array}$ & $54.2 \mathrm{c}$ & \begin{tabular}{|c|}
1.58 \\
$a b$
\end{tabular} & $1.5 \mathrm{ab}$ & \begin{tabular}{|c|}
$\begin{array}{c}0.337 d \\
e\end{array}$ \\
\end{tabular} & $\begin{array}{c}0.30 \\
\mathrm{~cd}\end{array}$ & \begin{tabular}{|c|}
26.3 \\
de
\end{tabular} & $24.0 \mathrm{fg}$ & $\begin{array}{c}12.84 a \\
b c\end{array}$ & $\begin{array}{c}12.95 a \\
b c\end{array}$ \\
\hline$M+J$ & $\begin{array}{c}57.63 \\
\text { bc }\end{array}$ & $55.0 \mathrm{c}$ & $\begin{array}{c}1.580 a \\
b\end{array}$ & $\begin{array}{c}1.46 \\
a b\end{array}$ & $\begin{array}{c}0.343 d \\
e\end{array}$ & $\begin{array}{c}0.317 \mathrm{c} \\
\mathrm{d}\end{array}$ & $\begin{array}{c}26.53 d \\
e\end{array}$ & $\begin{array}{c}24.17 f \\
g\end{array}$ & $\begin{array}{c}12.97 a \\
b c\end{array}$ & $\begin{array}{c}13.01 a \\
b\end{array}$ \\
\hline$M+F$ & $\begin{array}{c}58.16 \\
\text { bc }\end{array}$ & $55.8 \mathrm{c}$ & $\begin{array}{c}1.63 \\
a b\end{array}$ & $\begin{array}{c}1.55 \\
a b\end{array}$ & $0.347 d$ & $\begin{array}{c}0.320 c \\
d\end{array}$ & \begin{tabular}{|c|}
$26.63 d$ \\
$e$
\end{tabular} & $\begin{array}{c}24.37 e \\
f g\end{array}$ & $\begin{array}{c}13.10 \\
a b \\
\end{array}$ & $\begin{array}{c}13.17 a \\
b\end{array}$ \\
\hline $\mathbf{J}$ & $\begin{array}{c}64.83 \\
\mathrm{a}\end{array}$ & $\begin{array}{c}57.3 \\
\text { bc }\end{array}$ & $\begin{array}{c}1.69 \\
a b\end{array}$ & $\begin{array}{c}1.56 \\
a b\end{array}$ & $0.417 a$ & $\begin{array}{c}0.333 b \\
c\end{array}$ & $\begin{array}{c}29.06 c \\
\text { de }\end{array}$ & $\begin{array}{c}26.13 d \\
-g\end{array}$ & $14.37 \mathrm{a}$ & $\begin{array}{c}12.78 a \\
b c\end{array}$ \\
\hline$F$ & $\begin{array}{l}60.4 \\
a b c\end{array}$ & $61.97 \mathrm{a}$ & $\begin{array}{c}1.66 \\
a b\end{array}$ & $1.62 \mathrm{a}$ & $\begin{array}{c}0.36 \\
\mathrm{~cd}\end{array}$ & $0.38 \mathrm{a}$ & $\begin{array}{c}29.2 \\
\text { bc }\end{array}$ & $\begin{array}{c}26.93 d \\
-g\end{array}$ & $\begin{array}{c}12.44 \\
a-d\end{array}$ & $14.42 \mathrm{a}$ \\
\hline C & $\begin{array}{c}56.36 \\
\text { bc }\end{array}$ & $53.97 \mathrm{c}$ & $\begin{array}{c}1.53 \\
a b\end{array}$ & $1.40 \mathrm{~b}$ & $0.303 e$ & $0.283 d$ & $24.46 \mathrm{e}$ & $22.3 \mathrm{~g}$ & $\begin{array}{c}12.05 \\
a-d\end{array}$ & $\begin{array}{c}12.55 a \\
-d\end{array}$ \\
\hline
\end{tabular}

The results presented in Table 3 showed an increase in chlorophyll content of leaf as a result of treatment with Jisamar and Furdos synthetic biostimulants as will as spray with PGPR biostimulants. The first season results showed significant increments in chlorophyll a resulted from spray with each of $A$. brasilense, $B$. polymyxa and $P$. fluorescens alone, while, the mixture of them did not give significant difference. However, a significant increase was observed due to application with Furdose compared to control. 
The treatment with $P$. fluorescens + Jisamar recorded 5.04 and $4.89 \mathrm{mg} / \mathrm{cm}^{2}$ at first and second seasons, respectively. The trend of the second season results was similar to that of the first one. The spray with PGPR bacteria as will as PGPR with $\mathrm{F}$ or $\mathrm{J}$ potentially increased fruit production per tree, The spray with Azospirillum brasilense culture gave the highest results for both studied seasons, which attained 45.16 and $41.20 \mathrm{~kg} / \mathrm{tree}$ at 2009 and 2010 seasons, respectively, followed by the spray with Azospirillum brasilense + Jisamar which achieved production averaged 43.86 and $40.73 \mathrm{~kg} /$ tree for both seasons compared to 33.53 and $32.07 \mathrm{~kg} /$ tree for control treatment at the seasons 2009 and 2010, respectively. The differences than control were significant. Likewise, number of fruits per tree was positively affected by the different spray treatments, although, the most differences were not significant except the treatment of spray with Azospirillum brasilense or Azospirillum brasilense with $\mathrm{F}$ or $\mathrm{J}$ as will as $B$. polymyxa or $B$. polymyxa with $\mathrm{F}$ or $\mathrm{J}$ which attained significant differences at both seasons. The treatment of Azospirillum brasilense spray recorded the highest fruit number / tree at 2009 and 2010 seasons (346.3 and 314.0, respectively).

The increase of leaves chlorophyll content resulted from spray with synthetic or bacterial biostimulants is due to the stimulation and nutrientive effect on the plant. Gisamar and Furdose contains nutrients and stimulating compounds like short chain amino acids, plant phytohormones, vitamins and some macro- and micro-elements. Maini (2000) reported that the biostimulants (Siapton) which based on short chain amino acids increased the chlorophyll content for wheat plant. Also, Ferrini and Nicese (2002) sprayed Oak plants with two types of biostimulants; WP ${ }^{\text {th }}$ which composed of humic acid, seaweeds extract, Yucca extracts, vitamins and nitrogen fixing bacteria, and DP ${ }^{\mathrm{TM}}$ compound which composed of indo and ecto mycorrhiza, biostimulant bacteria, humic acids, carbohydrate, vitamins and Yucca extract. They claimed that photosynthesis and chlorophyll content were enhanced. The spray with Furdose and Jisamar, in the present study, stimulated the growth and increased fruit yield, but the spray with bacterial biostimulants had a strong influence than the synthetic biostimulants, and the treatment of Azospirillum brasilense foliar spray was the best in this concern. The mechanisms of bacterial biostimulants action may be summarized as follow:

1- Increase of number and size of plant cells due to the function of plant phytohormones like IAA, cytokinens and gebrillins (lqbal et al., 2011).

2- The polyamines contained in the synthetic biostimulants and these released by bacterial biostimulants regulate many growth processes; differentiation, formation of embryo, setting and ripening of fruits (Taha and Eid, 2011).

3- Stimulate production of antioxidants, therefore, decrease free radicals or reactive oxygen molecules leads to improvement of plant growth especially at biotic and abiotic stress like salinity, dryness and high exposure to UV. Most importantly, they provide essential information on cellular redox state, and regulate gene expression 
associated with biotic and abiotic stress responses to optimize defense and survival (Shao et al., 2008).

4- These stimulants contained some macro and micro-elements which enhance plant growth (Maini, 2000).

5- The bacterial biostimulants release plant phytohormones, amino acids, antioxidants, siderofores and antibiotics in addition to fixing atmospheric nitrogen in the rhizosphere or phyllosphere (MartinezViveros et al., 2010).

Table 3: Effect of spray with some synthetic and some bacterial biostimulants (PGPR cultures) on chlorophyll content and fruit yield of "Anna" apple trees

\begin{tabular}{|c|c|c|c|c|c|c|c|c|c|c|}
\hline \multirow[t]{2}{*}{ Treatments } & \multicolumn{4}{|c|}{\begin{tabular}{|c|c|}
$\underset{\left(\mathrm{mg} / \mathrm{cm}^{2}\right)}{\text { Chlorophyll a }}$ & Chlorophyll b \\
$\left(\mathrm{mg} / \mathrm{cm}^{2}\right)$
\end{tabular}} & \multicolumn{2}{|c|}{$\begin{array}{c}\text { Total } \\
\text { chlorophyll } \\
\left(\mathrm{mg} / \mathrm{cm}^{2}\right)\end{array}$} & \multicolumn{2}{|c|}{$\begin{array}{c}\text { Fruit weight } \\
(\mathrm{Kg} / \mathrm{tree})\end{array}$} & \multicolumn{2}{|c|}{ Fruit number } \\
\hline & 2009 & 2010 & 2009 & 2010 & 2009 & 2010 & 2009 & 2010 & 2009 & 2010 \\
\hline \multirow[t]{2}{*}{ A } & 4.64 & 4.44 & 2.56 & 2.42 & 7.20 & 6.86 & 45.16 & 41.20 & $346.3 a$ & 314.0 \\
\hline & & $\mathrm{cd}$ & $a b$ & $a b$ & $\mathrm{bc}$ & cde & $a$ & $a$ & & \\
\hline$A+J$ & $\begin{array}{l}4.69 \\
\mathrm{bcd}\end{array}$ & $\begin{array}{c}4.52 \\
b c\end{array}$ & $\begin{array}{c}2.51 \\
a b\end{array}$ & $\begin{array}{c}2.39 \\
a b\end{array}$ & $\begin{array}{c}7.20 \\
b c\end{array}$ & $\begin{array}{l}6.91 \\
\text { bcd }\end{array}$ & $\begin{array}{c}43.86 \\
a b\end{array}$ & $\begin{array}{c}40.73 \\
a b\end{array}$ & $\begin{array}{c}330.0 \\
a b\end{array}$ & $\begin{array}{c}300.3 \\
a b c\end{array}$ \\
\hline$A+F$ & $\begin{array}{c}4.56 \\
\mathrm{de}\end{array}$ & $\begin{array}{l}4.13 \\
\text { cde }\end{array}$ & $\begin{array}{l}2.47 \\
a b c\end{array}$ & $\begin{array}{l}2.37 \\
a b c\end{array}$ & $\begin{array}{c}7.03 \\
\mathrm{~cd}\end{array}$ & $\begin{array}{l}6.76 \\
\text { def }\end{array}$ & $\begin{array}{c}42.46 \\
a b c\end{array}$ & $\begin{array}{c}39.10 \\
a b c\end{array}$ & $\begin{array}{c}312.0 \\
a b c\end{array}$ & $\begin{array}{c}274.3 \\
\text { cde }\end{array}$ \\
\hline B & $\begin{array}{c}4.407 \\
d-a\end{array}$ & $\begin{array}{c}4.23 \\
\mathrm{de}\end{array}$ & $\begin{array}{l}2.32 \\
\text { bcd }\end{array}$ & $\begin{array}{l}2.20 \\
\text { bcd }\end{array}$ & $\begin{array}{l}6.73 \\
\text { def }\end{array}$ & $\begin{array}{c}6.43 \\
\mathrm{fg}\end{array}$ & $\begin{array}{c}42.96 \\
a b\end{array}$ & $\begin{array}{c}39.40 \\
a b c\end{array}$ & $\begin{array}{c}327.6 \\
a b\end{array}$ & $\begin{array}{c}298.0 \\
\text { a-d }\end{array}$ \\
\hline$B+J$ & $\begin{array}{c}4.55 \\
\mathrm{de}\end{array}$ & $\begin{array}{l}4.32 \\
\text { cde }\end{array}$ & $\begin{array}{l}2.29 \\
\text { bcd }\end{array}$ & $\begin{array}{l}2.18 \\
\text { bcd }\end{array}$ & $\begin{array}{c}6.08 \\
c-f\end{array}$ & $\begin{array}{c}6.50 \\
\mathrm{fg}\end{array}$ & $\begin{array}{c}40.93 \\
\text { a-d }\end{array}$ & $\begin{array}{c}37.53 \\
\text { a-d }\end{array}$ & $\begin{array}{c}318.3 \\
a b c\end{array}$ & $\begin{array}{c}295.3 \\
\text { a-d }\end{array}$ \\
\hline$B+F$ & $\begin{array}{c}4.44 \\
\text { def }\end{array}$ & $\begin{array}{l}4.25 \\
\text { de }\end{array}$ & $\begin{array}{c}2.39 \\
a-d\end{array}$ & $\begin{array}{c}2.29 \\
a-d\end{array}$ & $\begin{array}{c}6.84 \\
\text { C-f }\end{array}$ & $\begin{array}{l}6.54 \\
\text { efg }\end{array}$ & $\begin{array}{c}40.83 \\
a-d\end{array}$ & $\begin{array}{c}37.23 \\
\text { a-d }\end{array}$ & $\begin{array}{c}328.0 \\
a b\end{array}$ & $\begin{array}{c}304.6 \\
a b\end{array}$ \\
\hline $\mathbf{P}$ & $\begin{array}{l}4.91 \\
a b c\end{array}$ & $\begin{array}{c}4.72 \\
a b\end{array}$ & $2.61 \mathrm{a}$ & $2.49 \mathrm{a}$ & $\begin{array}{c}7.53 \\
a b\end{array}$ & $\begin{array}{c}7.21 \\
a b\end{array}$ & $\begin{array}{c}39.23 b \\
c d\end{array}$ & $\mid \begin{array}{c}36.10 b \\
-e\end{array}$ & $\begin{array}{c}316.6 \mathrm{a} \\
\mathrm{bc}\end{array}$ & $\begin{array}{c}283.3 b \\
-e\end{array}$ \\
\hline P+J & $5.04 \mathrm{a}$ & $4.89 \mathrm{a}$ & $2.60 \mathrm{a}$ & $2.49 \mathrm{a}$ & $7.66 \mathrm{a}$ & $7.39 \mathrm{a}$ & $\begin{array}{l}37.4 \\
\text { cde }\end{array}$ & $\begin{array}{c}34.97 c \\
-f\end{array}$ & $c \begin{array}{c}306.3 \mathrm{~b} \\
\mathrm{c}\end{array}$ & $\begin{array}{c}278.0 \\
b-e\end{array}$ \\
\hline $\mathrm{P}+\mathrm{F}$ & $\begin{array}{c}4.93 \\
a b\end{array}$ & $\begin{array}{c}4.73 \\
a b\end{array}$ & $\begin{array}{c}2.53 \\
a b\end{array}$ & $\begin{array}{c}2.42 \\
\text { ab }\end{array}$ & $\begin{array}{c}7.47 \\
a b\end{array}$ & $\begin{array}{l}7.15 \\
a b c\end{array}$ & $\begin{array}{c}37.03 c \\
d e\end{array}$ & $\begin{array}{c}33.93 \mathrm{~d} \\
\text { ef }\end{array}$ & $\mathrm{d} 321.0 \mathrm{a}$ & $\begin{array}{c}296.0 a \\
-d\end{array}$ \\
\hline $\mathbf{M}$ & $4.15 \mathrm{~g}$ & $3.96 \mathrm{f}$ & $2.16 \mathrm{~d}$ & $2.05 \mathrm{~d}$ & $6.31 \mathrm{~g}$ & $6.01 \mathrm{~h}$ & $33.43 \mathrm{e}$ & $30.50 \mathrm{f}$ & $f \mid 287.6 \mathrm{c}$ & $263.3 \mathrm{e}$ \\
\hline $\mathrm{M}+\mathrm{J}$ & $\begin{array}{c}4.41 \mathrm{~d}- \\
\mathrm{g}\end{array}$ & 4.16 ef & $\begin{array}{c}2.20 \\
\mathrm{~cd}\end{array}$ & $2.09 \mathrm{~d}$ & $\begin{array}{l}6.62 \\
\text { efg }\end{array}$ & $\begin{array}{c}6.25 \\
\mathrm{gh}\end{array}$ & $\begin{array}{c}37.23 c \\
\text { de }\end{array}$ & $c \begin{array}{c}34.37 c \\
-f\end{array}$ & $c \begin{array}{c}300.6 b \\
c\end{array}$ & 262.0 \\
\hline \multirow[t]{2}{*}{$\mathrm{M}+\mathrm{F}$} & $4.25 \mathrm{fg}$ & $4.07 \mathrm{ef}$ & 2.32 & 2.19 & $6.57 \mathrm{fg}$ & 6.26 & $38.5 b-$ & $-36.20 b$ & b303.6b & 269.7 \\
\hline & & & bcd & bcd & & gh & $\frac{\mathrm{e}}{7 \sqrt{n d}}$ & $-e$ & c & \\
\hline $\mathrm{J}$ & $\begin{array}{l}4.89 \\
a b c\end{array}$ & $4.17 \mathrm{ef}$ & $\begin{array}{c}2.53 \\
a b\end{array}$ & $\begin{array}{c}2.41 \\
a b\end{array}$ & $\begin{array}{c}7.42 \\
a b\end{array}$ & $\begin{array}{c}6.59 d- \\
q\end{array}$ & $\begin{array}{c}1-\begin{array}{c}37.4 c d \\
e\end{array} \\
e\end{array}$ & $\left|\begin{array}{c}33.27 d \\
\text { ef }\end{array}\right|$ & $\begin{array}{c}315.0 \mathrm{a} \\
\mathrm{bc}\end{array}$ & $\begin{array}{l}285.6 \mathrm{c} \\
-\mathrm{e}\end{array}$ \\
\hline \multirow[t]{2}{*}{$\mathbf{F}$} & $4.427 \mathrm{~d}$ & 4.71 & 2.56 & 2.41 & 6.93 & 7.11 & $36.53 d$ & $34.77 \mathrm{c}$ & $\mathrm{c} 313.3 \mathrm{a}$ & 287.6 \\
\hline & $-g$ & $a b$ & $a b$ & $a b$ & cde & $a b c$ & $\mathrm{e}$ & $-f$ & $b c$ & \\
\hline \multirow[t]{2}{*}{ C } & 4.29 & $4.09 \mathrm{ef}$ & $2.230 c$ & 2.14 & $6.52 \mathrm{fg}$ & 6.23 & 33.5 & $32.07 \mathrm{e}$ & $\mathrm{e} 292.0$ & $266.0 \mathrm{e}$ \\
\hline & efg & & d & $\mathrm{cd}$ & & gh & & $f$ & & \\
\hline
\end{tabular}

Data of Table 4, some important quality parameters of the fruits were determined like soluble solid content (SSC), acidity, firmness and color. The 
values of SSC through the two studying seasons were generally lowered due to spraying with stimulants whether were synthetic or biological, but the differences from the control did not usually reach to significance. While acidity of the fruits, in general, increased by the sprayed treatments especially with bacterial biostimulants in both seasons. The foliar spray with the studied bacterial biostimulants, generally, led to significant increases in firmness values of the fruit, at the second season only. The treatments of synthetic or biological decreased the color degree of the fruits and this effect was clear for results of the second season where all differences than control treatment were significant. The control values recorded 94.84 and 90.34 for seasons 2009 and 2010 compared to values ranged from 65.02 to 83.84 at season 2009 and from 70.63 to 82.25 at season 2010 .

Table 4: Effect of spray with some synthetic and some bacterial biostimulants (PGPR cultures) on some fruit quality characters of "Anna" apple trees

\begin{tabular}{|l|c|c|c|c|c|c|c|c|}
\hline \multirow{2}{*}{ Treatments } & \multicolumn{2}{|c}{ SSC } & \multicolumn{2}{c|}{ Acidity } & \multicolumn{2}{c|}{ Firmness } & \multicolumn{2}{c|}{ Color } \\
\cline { 2 - 9 } & $\mathbf{2 0 0 9}$ & $\mathbf{2 0 1 0}$ & $\mathbf{2 0 0 9}$ & $\mathbf{2 0 1 0}$ & $\mathbf{2 0 0 9}$ & $\mathbf{2 0 1 0}$ & $\mathbf{2 0 0 9}$ & $\mathbf{2 0 1 0}$ \\
\hline $\mathbf{A}$ & $13.20 \mathrm{a}$ & $13.33 \mathrm{~J}$ & $0.82 \mathrm{ab}$ & $0.86 \mathrm{bc}$ & $226.0 \mathrm{ab}$ & $263.3 \mathrm{~b}$ & $79.23 \mathrm{a}$ & $77.68 \mathrm{bcd}$ \\
\hline $\mathbf{A + F}$ & $12.93 \mathrm{abc}$ & $13.16 \mathrm{ab}$ & $0.85 \mathrm{~b}$ & $0.86 \mathrm{bc}$ & $260.0 \mathrm{a}$ & $263.0 \mathrm{~b}$ & $74.17 \mathrm{a}$ & $75.01 \mathrm{bcd}$ \\
\hline $\mathbf{B}$ & $13.06 \mathrm{ab}$ & $13.26 \mathrm{a}$ & $0.84 \mathrm{~b}$ & $0.87 \mathrm{c}$ & $237.6 \mathrm{a}$ & $245.3 \mathrm{c}$ & $76.17 \mathrm{a}$ & $77.85 \mathrm{bcd}$ \\
\hline $\mathbf{B + J}$ & $12.60 \mathrm{bcd}$ & $12.98 \mathrm{abc}$ & $0.82 \mathrm{bc}$ & $0.78 \mathrm{ef}$ & $267.6 \mathrm{a}$ & $280.0 \mathrm{a}$ & $73.02 \mathrm{a}$ & $75.62 \mathrm{bcd}$ \\
\hline $\mathbf{B + F}$ & $12.40 \mathrm{~cd}$ & $12.26 \mathrm{~d}$ & $0.94 \mathrm{a}$ & $0.92 \mathrm{ab}$ & $215.6 \mathrm{ab}$ & $285.6 \mathrm{a}$ & $72.96 \mathrm{a}$ & $75.37 \mathrm{bcd}$ \\
\hline $\mathbf{P}$ & $12.23 \mathrm{~d}$ & $12.83 \mathrm{abc}$ & $0.94 \mathrm{a}$ & $0.93 \mathrm{ab}$ & $280.0 \mathrm{a}$ & $283.6 \mathrm{a}$ & $71.11 \mathrm{a}$ & $75.26 \mathrm{bcd}$ \\
\hline $\mathbf{P + J}$ & $12.43 \mathrm{~cd}$ & $12.63 \mathrm{bcd}$ & $0.84 \mathrm{~b}$ & $0.86 \mathrm{bcd}$ & $269.0 \mathrm{a}$ & $277.6 \mathrm{a}$ & $72.10 \mathrm{a}$ & $75.51 \mathrm{bcd}$ \\
\hline $\mathbf{P + F}$ & $12.40 \mathrm{~cd}$ & $12.66 \mathrm{bcd}$ & $0.82 \mathrm{bc}$ & $0.77 \mathrm{ef}$ & $286.3 \mathrm{a}$ & $284.6 \mathrm{a}$ & $65.02 \mathrm{a}$ & $70.63 \mathrm{~d}$ \\
\hline $\mathbf{M}$ & $12.43 \mathrm{~cd}$ & $12.63 \mathrm{bcd}$ & $0.97 \mathrm{a}$ & $0.96 \mathrm{a}$ & $286.3 \mathrm{a}$ & $286.3 \mathrm{a}$ & $66.29 \mathrm{a}$ & $70.26 \mathrm{~d}$ \\
\hline $\mathbf{M + J}$ & $13.13 \mathrm{ab}$ & $13.30 \mathrm{a}$ & $0.80 \mathrm{bc}$ & $0.77 \mathrm{ef}$ & $221.0 \mathrm{ab}$ & $229.0 \mathrm{~d}$ & $71.81 \mathrm{a}$ & $73.84 \mathrm{~cd}$ \\
\hline $\mathbf{M + F}$ & $12.43 \mathrm{~cd}$ & $12.60 \mathrm{~cd}$ & $0.82 \mathrm{bc}$ & $0.81 \mathrm{cde}$ & $221.0 \mathrm{ab}$ & $227.0 \mathrm{~d}$ & $73.73 \mathrm{a}$ & $75.32 \mathrm{bcd}$ \\
\hline $\mathbf{J}$ & $12.60 \mathrm{bcd}$ & $12.86 \mathrm{abc}$ & $0.81 \mathrm{bc}$ & $0.79 \mathrm{def}$ & $220.0 \mathrm{ab}$ & $229.0 \mathrm{~d}$ & $73.83 \mathrm{a}$ & $75.29 \mathrm{bcd}$ \\
\hline $\mathbf{F}$ & $12.66 \mathrm{a}-\mathrm{d}$ & $12.83 \mathrm{abc}$ & $0.74 \mathrm{~cd}$ & $0.72 \mathrm{fg}$ & $222.0 \mathrm{ab}$ & $232.6 \mathrm{~cd}$ & $83.84 \mathrm{a}$ & $81.54 \mathrm{bc}$ \\
\hline $\mathbf{C}$ & $12.88 \mathrm{abc}$ & $13.00 \mathrm{abc}$ & $0.74 \mathrm{~cd}$ & $0.70 \mathrm{~g}$ & $223.0 \mathrm{ab}$ & $234.6 \mathrm{~cd}$ & $82.30 \mathrm{a}$ & $82.25 \mathrm{~b}$ \\
\hline \multirow{2}{*}{$\mathbf{n}$} & $13.06 \mathrm{~b}$ & $13.03 \mathrm{abc}$ & $0.69 \mathrm{~d}$ & $0.77 \mathrm{ef}$ & $207.0 \mathrm{ab}$ & $220.6 \mathrm{~d}$ & $94.84 \mathrm{a}$ & $90.34 \mathrm{a}$ \\
\hline
\end{tabular}

*J: Jisamar; F: Furdos; A: Azospirillum brasilense; B: Bacillus polymyxa; P: Pseudomonas fluorescens; M: microbial mixture.

${ }^{\star \star}$ Means with different letters within the same column differ significantly at $\mathbf{P}<0.05$.

The spray with gibberellic acid $\left(\mathrm{GA}_{3}\right)$ showed improvement for fruit quality of jambu madu (Moneruzzaman et al, 2011), apple fruits (Ryu et al., 2011). At the same time Pirlak and Köse (2009) claimed that the spray of strawberry with Pseudomonas BA-8 and other PGPR bacteria increased TSS and acidity of the fruits. The spray with synthetic or bacterial biostimulants lead to decrease of SSC values and color of fruits but increased acidity and firmness, however, most variations were not significant. Sahain et al. (2007) reported different results, whereas, they showed that spray of apple trees with EM biostimulant (composed of fluorescent bacteria and fermentative fungi) increased TSS and decreased acidity and firmness of the fruits, the difference in the influence may be due to the variation in the microbial composition between EM and the present used PGPR bacteria. Results of 


\section{Nour El-Din et al.}

Esitken et al. (2009) were similar to our findings as they sprayed the trees with some PGPR types.

Viable counts (Table 5) of the used microorganisms as foliar spray notably increased in the phyllosphere of the plant. The counts of Azospirillum brasilense appeared very high due to spray with the liquid culture of Azospirillum brasilense, this effect clearly appeared after spray with Azospirillum brasilense alone or mixing with Jismar or Furdos. The spray with the mixture of Azospirillum brasilense and Jismar attained the highest average number of Azospirillum evaluated $240 \times 10^{2} \mathrm{cfu} / \mathrm{cm}^{2}$ of leaf. Likewise, the spray with $B$. polymyxa culture alone or mixed with Jisamar had potential effect in increasing its numbers in the phyllosphere, which represented $45 \mathrm{x}$ $10^{2}$ and $40 \times 10^{2} \mathrm{cfu} / \mathrm{cm}^{2}$ of leaf, respectively. Similar results happened with the spray with $P$. fluorescens which caused clear increase of the applied microbe in the phyllosphere area, the counts were $5 \times 10^{2}, 30 \times 10^{2}$ and $25 \times$ $10^{2} \mathrm{cfu} / \mathrm{cm}^{2}$ of leaf due to spray with $P$. fluorescens only, $P$. fluorescens $+J$ and $P$. fluorescens $+F$, respectively. The spray with the mixture of the three microorganisms only or mixed with $\mathrm{J}$ or $\mathrm{F}$ attained increments over that of the control, but these increments were lower than these caused by spray with each microbe alone. The counts of the tested baceria of control treatment averaged $0.1 \times 10^{2}, 0.05 \times 10^{2}$ and $0.04 \times 10^{2} \mathrm{cfu} / \mathrm{cm}^{2}$ of leaf due to the spraying with Azospirillum brasilense, B. polymyxa and $P$. fluorescenc, respectively.

Table 5: Effect of spray with some synthetic and some bacterial biostimulants (PGPR cultures) on the counts of the used PGPR bacterial cells in the phyllosphere of "Anna" apple trees at 2010 season.

\begin{tabular}{|c|c|c|c|}
\hline Treatment & Azospirillum $\times 10^{2}$ & B. polymyxa $\times 10^{2}$ & P. fluorescens $\times 10^{2}$ \\
\hline $\mathbf{A}$ & 34.0 & 0.1 & $\begin{array}{c}0.4 \\
\end{array}$ \\
\hline $\mathbf{A + J}$ & 240.0 & 0.2 & 0.3 \\
\hline$A+F$ & 4.0 & 0.1 & 0.3 \\
\hline B & 0.2 & 45.0 & 0.3 \\
\hline $\mathbf{B + J}$ & 0.2 & 40.0 & 0.5 \\
\hline$B+F$ & 0.2 & 6.0 & 0.5 \\
\hline $\mathbf{P}$ & 0.3 & 0.2 & 5.0 \\
\hline $\mathbf{P + J}$ & 0.3 & 0.11 & 30.0 \\
\hline $\mathbf{P + F}$ & 0.5 & 0.3 & 25.0 \\
\hline $\mathbf{M}$ & 2.0 & 0.3 & 0.5 \\
\hline $\mathbf{M + J}$ & 5.0 & 2.0 & 0.3 \\
\hline$M+F$ & 7.0 & 0.4 & 1.0 \\
\hline $\mathbf{J}$ & 0.5 & 0.02 & 0.05 \\
\hline $\mathbf{F}$ & 1.0 & 0.4 & 1.0 \\
\hline $\mathbf{C}$ & 0.1 & 0.05 & 0.04 \\
\hline
\end{tabular}

Pseudomonas fluorescens; M: microbial mixture.

Spray of plant phyllosphere with PGPR bacteria resulted in increased counts of these bacteria in the phyllosphere, our results confirmed those of Kim et al. (2011) as they reported effective colonization of the used PGPR 
bacteria in the phyllosphere of pepper plants compared to control plants. They, also, noted that most of the fluorescent bacterial cells were localized near the stomata and throughout the blade part of the inoculated leaves.

Readings of Table 6 show the productivity of apple fruits (tons/fed.), costs of production, total and net return (LE/fed). The fixed costs (land rent, labors, fertilizers, pesticides, hoeing and pruning) reached about $5000 £$ E/fed. Changed costs include chemical or biological stimulators, rent of spray machine and spray labor reached $600 £ \mathrm{E} / \mathrm{fed}$. The price of apple fruits evaluated $3500 £ \mathrm{E} /$ ton.

Table 6: Economic evaluation of apple trees spray with some synthetic and some bacterial biostimulants (PGPR cultures)

\begin{tabular}{|c|c|c|c|c|c|c|c|}
\hline Treatments & $\begin{array}{c}\text { Fixed } \\
\text { costs } \\
(£ . E / \text { fed. })\end{array}$ & $\begin{array}{l}\text { Changed } \\
\text { costs } \\
\text { (£.E/fed.) }\end{array}$ & $\begin{array}{c}\text { Total } \\
\text { costs } \\
\text { (£.E/fed.) }\end{array}$ & $\begin{array}{l}\text { Total } \\
\text { yield } \\
\text { (Ton/ } \\
\text { fed.) }\end{array}$ & $\begin{array}{c}\text { Value } \\
\text { (£.E/fed.) }\end{array}$ & $\begin{array}{l}\text { Net } \\
\text { return } \\
\text { (E.E/ } \\
\text { fed.) }\end{array}$ & $\begin{array}{c}\text { Increase / } \\
\text { decrease } \\
\text { in return } \\
\text { than } \\
\text { control } \\
\end{array}$ \\
\hline $\mathbf{A}$ & 5000 & 600 & 5600 & 7.77 & 27195 & 21595 & 5945 \\
\hline$A+J$ & 5000 & 600 & 5600 & 7.61 & 26635 & 21035 & 5385 \\
\hline$A+F$ & 5000 & 600 & 5600 & 7.34 & 25690 & 20090 & 4440 \\
\hline B & 5000 & 600 & 5600 & 7.41 & 25935 & 20335 & 4685 \\
\hline $\mathbf{B + J}$ & 5000 & 600 & 5600 & 7.06 & 24710 & 19110 & 3460 \\
\hline$B+F$ & 5000 & 600 & 5600 & 7.03 & 24605 & 19005 & 3355 \\
\hline $\mathbf{P}$ & 5000 & 600 & 5600 & 6.78 & 23730 & 18130 & 2480 \\
\hline$P+J$ & 5000 & 600 & 5600 & 6.51 & 22785 & 17185 & 1535 \\
\hline $\mathrm{P}+\mathrm{F}$ & 5000 & 600 & 5600 & 6.39 & 22365 & 16765 & 1115 \\
\hline M & 5000 & 600 & 5600 & 5.75 & 20125 & 14525 & -1125 \\
\hline $\mathbf{M + J}$ & 5000 & 600 & 5600 & 6.44 & 22540 & 16940 & 1290 \\
\hline $\mathbf{M}+\mathbf{F}$ & 5000 & 600 & 5600 & 6.72 & 23520 & 17920 & 2270 \\
\hline $\mathbf{J}$ & 5000 & 600 & 5600 & 6.36 & 22260 & 16660 & 1010 \\
\hline $\mathbf{F}$ & 5000 & 600 & 5600 & 6.42 & 22470 & 16870 & 1220 \\
\hline C & 5000 & 0.0 & 5000 & 5.90 & 20650 & 15650 & - \\
\hline
\end{tabular}

J: Jisamar; F: Furdos; A: Azospirillum brasilense; B: Bacillus polymyxa; P: Pseudomonas fluorescens; M: microbial mixture.

The calculations of net return per feddan exhibited that the application of treatments of Jisamar and Furdose attained net return over the treatment of control by about 1010 and $1220 £ \mathrm{E} / \mathrm{fed}$, respectively. The application of bacterial biostimulants alone or mixed with Jisamar or Furdose, however, attained net return much higher than both the control and synthetic stimulators. The highest obtained increase in net return over control treatment was attained by spray with Azospirillum brasilense (5945 £ E/fed) followed by treatment of Azospirillum brasilense $+\mathrm{J}$ which achieved $5385 \mathrm{E}$ E/fed. An increase reached $4685 £$ E/fed due to spray with $B$. polymixa. Otherwise, the only treatment that exhibited decrease than control was the spray with the mixture of the microorganisms.

Finally, from the economic evaluation for the influence of synthetic and bacterial biostimulants, it could be conclude that these compounds 


\section{Nour El-Din et al.}

increased plant growth and yield without a notable increase in costs because of the lower price of these compounds. Thus, the net return was positive, whereas, the spray with Azospirillum brasilense liquid culture gave the highest net return (5945 £ E/feddan), and the net return of bacterial biostimulants was much larger than those of synthetic biostimulants. Therefore, we recommend the spray of apple trees twice with liquid cultures of effective Azospirillum brasilense cultures by the rate of $20 \mathrm{~L}$ per feddan as alternative to the application of synthetic biostimulants.

Table (7): Production of indole acetic acid (IAA) and total gibberellins by the used bacterial strains.

\begin{tabular}{|l|c|c|}
\hline \multirow{2}{*}{ Strain } & \multicolumn{2}{|c|}{ G.P.S $^{\star}(\mathbf{m g} / \mathbf{l})$} \\
\cline { 2 - 3 } & IAA & GA \\
\hline Pseudomonas fluorescense & 160.0 & 485.0 \\
\hline Bacillus polymyxa & 157.0 & 574.5 \\
\hline Azospirillum brasilense & 101.4 & 604.3 \\
\hline
\end{tabular}

G.P.S. ${ }^{*}$ Growth Promoting Substances

The different bacterial isolates produced different amounts of IAA and GA (Table 7). However, Azospirillum brasilense release the highest amounts of GA $(604.3 \mathrm{mg} / \mathrm{l})$ compared to 485 and $574.5 \mathrm{mg} / \mathrm{l}$ for Pseudomonas fluorescence and Bacillus polymyxa respectively. Pseudomonas fluorescence attained the highest IAA amount (160 mg/l) compared to 157 and $101.4 \mathrm{mg} / \mathrm{l}$ for Bacillus polymyxa and Azospirillum brasilense, respectively.

Yicheng Tu (2000) reviewed that gibberellins(GA) were first discovered by Japanese scientists studying a rice disease characterized by excessive stem elongation named "bakenae" (foolish seedling). The symptom was found to be stimulated by compound(s) released by a fungus Gibberella fujikuroi. In 1935, the compound was isolated and named after the fungus as "Gibberellin". Further experiments showed that GA not only occurred in plants but also played a key role in various aspects of plant growth and development. GAs were found to promote cell expansion and perhaps cell division that led to elongation of shoot tissues and development of a number of other morphological structures such as inflorescences and fruit.

The effects of plant growth promoting rhizobacteria (PGPR) on the rooting and root growth of semi-hardwood and hardwood kiwifruit stem cuttings were investigated by Martínez-Viveros (2010). The PGPR used were Bacillus RC23, Paenibacillus polymyxa RC05, Bacillus subtilis OSU142, Bacillus RC03, Comamonas acidovorans RC41, Bacillus megaterium RC01 and Bacillus simplex RC19. All the bacteria showed indole-3-acetic acid (IAA) producing capacity. Among the PGPR used, the highest rooting ratios were obtained at $47.50 \%$ for semi-hardwood stem cuttings from Bacillus RC03 and Bacillus simplex RC19 treatments and $42.50 \%$ for hardwood stem cuttings from Bacillus RC03. As well, Comamonas acidovorans RC41 inoculations indicated higher value than control treatments. The results suggest that these PGPR can be used in organic nursery material production and point to the feasibility of synthetic auxin (IBA) replacement by organic management based on PGPR. While Anwar (2007) found that Azospirillum K-I produced 
higher amount of $\mathrm{GA}(10 \mathrm{ug} / \mathrm{ml})$ which decreased in later growth stages. This decrease in GA might be due to its hydrolysis by bacterial strain. Azoarcus K$\mathrm{I}$ and Azospirillum ER-2 and ER- 20 also produced indole, acetamide as identified by HPLC. These strains were also useful for increasing rice biomass, N-uptake and fertilizer- $\mathrm{N}$ use efficiency. In green house experiments, inoculation of rice with PGPR increased chlorophyll, leaf area, tiller number, plant height, root shoot biomass and grain yield in rice. Soil, root, leaf and stem inoculation methods were equally useful for plant growth improvement

\section{CONCLUSION}

The spray of Anna apple trees with synthetic biostimulants (Jisamar and Furdose) resulted in an increase in growth and yield of apple trees and did not significantly affected the quality of the resulted fruits, but leads to increase of fruit weight. The spray with bacterial biostimulants, however, gave best results and the application of Azospirillum brasilense surpassed the other bacterial biostimulants. The single spray with bacterial biostimulant showed best results than their mixture with Jisamar or Furdose. Thus, the spray with Azospirillum brasilense was the best which may be safely used as an alternative to the chemical synthetic biostimulants.

\section{REFERENCES}

A.O.A.C. (1990). Official methods of analyses. $15^{\text {th }}$ Ed., Association of Official Analytical Chemists. Washington, DC, USA.

Allen, O. N. (1959). Experiments in soil Bacteriology. University of Wiscosin. $2^{\text {ed }}$ Ed., 202.

Anwar, G (2007). Production of growth hormones and nitrogenase by diazotrophic bacteria and their effect on plant growth. University of the Punjab, Lahore / Institute Of Biochemistry And Biotechnology

Bric, J. M.; R. M. Bostock and S. E. Silverstone (1991). Rapid in situ assay for indole acetic acid production by bacteria immobilized on nitrocellulose membrane. Appl. Environ. Microbiol. Biotechnol., 35: 646-650.

Difco Manual (1984). Dehydrated culture media and reagent for microbiology. Laboratories incorporated Detroit. Michigan, 48232 USA. p.1027

Döbereiner, J.; Marrial, L. E. and Nery, M. (1976). Ecological distribution of Spirillum lipoferum. Beijerink. Can. J. Microbiol., 22: 1464-1473.

Eissa, Fawzia, M. (2003). Effect of some biostimulants on vegetative growth, yield and fruit quality of "Kelsey" Plum. Egypt. J. Appl. Sci., 18:716-735.

Erturk, Y.; Cakmakci, R.; Duyar, O. and Turan, M. (2011). The effects of plant growth promoting rhizobacteria on vegetative growth and leaf nutrient contents of hazelnut seedlings (Turkish hazelnut cv, Tombul and Sivri). International Journal of Soil Science, 6: 188-198.

Esitken, A. (2011). Use of plant growth promoting rhizobacteria in horticultural crops. In Bacteria in Agrobiology: Crop Ecosystems, Springer Berlin Hiedelberg: 189-235. 


\section{Nour El-Din et al.}

Esitken, A.; Pirlak, L.; Ipek, M.; Donmez, M. F.; Cakmakci R. and Sahin, F. (2009). Fruit bio-thinning by plant growth promoting bacteria (PGPB) in apple cvs. Golden delicious and Braeburn. Biol. Agri. and Horti., 26: 379-390.

Esitkin, A.; Karlidag,H.; Ercisli, S.; Turan, M. and Sahin, F. (2004). The effect of spraying a growth promoting bacteria on the yield, growth and nutrient element composition of leaves of apricot (Prunus armeniaca $\mathrm{L}$. cv. Hacihaliloglu). Australian J. of Agr. Res., 54: 377-380.

Fathi, M.A.; Eissa, Fawzia, M. and Yehia, M. M. (2002). Improving growth, yield and fruit quality of "Desert red" peach and "Anna" apple by using some biostimulants. Minia J. of Agric. Res.\& Develop., 22: 519-534.

Ferree, D. C., and C. G. Forshey (1988). Influence of pruning and urea sprays on growth and fruiting of spur-bound 'Delicious' apple trees. J. Amer. Soc. Hort. Sci. 113 (5): 699-703.

Ferrini, F. and Nicese, F. P. (2002). Response of English oak (Quercus robur L.) trees to biostimulants application in the urban environment, $\mathrm{J}$. Arboriculture, 28: 70-75.

Glickmann, E. and Y. Dessoux (1995). A critical examination of the specificity of the salokowski reagent for indolic compounds produced by phytopathogenic bacteria. Appl. Environ. Microbiol., 61: 793-796.

Govidasamy, V., Senthilkumar, M., Gaikwad, K., Annapurna, K. (2008). Isolation and characterization of ACC deaminase gene from two plant growth-promoting rhizobacteria. Curr. Microbiol. 57, 312-317.

Harold, E. P. (1985) Evaluation of quality of fruits and vegetables. AVI publications - West Port. Comm., USA.

Hino, S. and Wilson, P.W. (1958). Nitrogen fixation by a facultative Bacillus, J. Bacteriol., 75: 413.

Iqbal, S.; Nazar, R.; lqbal R., Masood A. and Khan, N. A. (2011). Role of gibberellins in regulation of source - sink relations under optimal and limiting environmental conditions. Current Science, 100: 998-1007.

Karakurt, H. and Aslantas, R. (2010). Effect of some plant growth promoting Rhizobacteria (PGPR) strains on plant growth and leaf nutrient content of apple. Journal of Fruit and Ornamental Plant Research, 18: 101-110.

Kim, Y. S.; Park, K. and Sa, T. (2011). Foliar colonization and growth promotion of red pepper (Capsicum annuum L.) by Methylobacterium oryzae CBMB20. J. Appl. Biol. Chem. 54: 120-125.

King, E. O.; M. K. Ward and D. E. Raney (1954). Two simple media for the determination of pyocyanin and fluorscin. J. Lab. Med., 44:301-307.

Maini, F. (2000). The experience of the first biostimulants, based on amino acids and peptides: a short retrospective review on the laboratory researches the practical results. Fertilitas Agororum, 1:29-43.

Martínez-Viveros, O.; M.A. Jorquera, D.E. Crowley, G. Gajardo and M.L. Mora (2010). Mechanisms and practical considerations involved in plant growth promotion by rhizobacteria. J. Soil Sci. Plant Nutr. 10 (3): $293-319$.

McGuire, R. G. (1992). Reporting of objective color measurements. Hort. Sci. 27 (12):1254 - 1260. 
Moneruzzaman, K. M., Hossain, A. B. M. S., Normaniza, O. and Boyce, A. N. (2011). Growth, yield and quality responses to gibberellic acid $\left(\mathrm{GA}_{3}\right)$ of Wax apple Syzygium samarangense var. Jambu air madu fruits grown under field conditions. African Journal of Biotechnology Vol. 10(56), pp. 11911-11918.

Nour El-Din M. (2006). Influence of foliar application with some micronutrients and some microorganisms on growth of peanut plant. Alex. J. Agric. Res., 51: 113-119.

Oliveira, C.A., Alves, V.M.C., Marriel, I.E., Gomes, E.A., Scotti, M.R., Carneiro, N.P., Guimaraes, C.T., Schaffert, R.E., Sá, N.M.H. 2009. Phosphate solubilizing microorganisms isolated from rhizosphere of maize cultivated in an oxisol of the Brazilian Cerrado Biome. Soil Biol. Biochem. 41, 1782-1787.

Pırlak, L. \& Köse, M. (2009). Effects of Plant Growth Promoting Rhizobacteria on Yield and Some Fruit Properties of Strawberry. Journal of Plant Nutrition, 32: 1173-1184.

Rami, M. (1982). Formulae for determination of chlorophyllous pigments extracted with $N, N$ - dimethyl formamide. Plant Physiol. 69,1376-1381.

Rami, M. and D. Porath (1980). Chlorophyll determination in intact tissues using $N, N$ - dimethyl formamide. Plant Physiol. 65: 478-479.

Ryu, C.; Shin, J.; Qi, W.; Ruhong, M.; Kim, E. J. and Jae Gu Pan, J. G. (2011). Potential for augmentation of fruit quality by foliar application of bacilli spores on apple tree. Plant Pathol. J. 27: 164-169.

Sahain, M. F. M.; Elham Z. Abd El Motty,.El- Shiekh, M. H. and Laila. F. Hagagg (2007). Effect of some biostimulant on growth and fruiting of anna apple trees in newly reclaimed areas. Res. J. of Agric. and Biol. Sci., 3: 422-429.

Saharan, B.S. and Nehra, V. (2011). Plant Growth Promoting Rhizobacteria: A Critical Review. Life Sciences and Medicine Research, LSMR-21: 130.

Shao, H. P.; Chu, L. Y.; Lu, Z. and Kang C. (2008). Primary antioxidant free radical scavenging and redox signaling pathways in higher plant cells. Int. J. Biol. Sci., 4:8-14.

Spaepen, S., Dobbelaere, S., Croonenborghs, A., Vanderleyden, J. (2008). Effects of Azospirillum brasilense indole-3-acetic acid production on inoculated wheat plants. Plant Soil 312, 15-23.

Spinelli, F.; Fiori, G.; Noferini, M.; Sprocatti, M. and Costa, G. (2010). A novel type of seaweed extract as a natural alternative to the use of iron chelates in strawberry production. Scientia Horticulturae, 125, 263-269.

Steel, R. G. and Torrie, J. H. (1980) Principles and procedures of statistics. Abiometrical approach. MC Grow hill, New York.

Taha L.S. and Eid, R.A. (2011). Stimulation effect of some bioregulators on flowering, chemical constituents, essential oil and phytohormones of tuberose (Polianthes tuberos L.). Journal of American Science; 7: 165 171.

Udagwa, K. and S. Kinoshita (1961). A colorimetric determination of gibberellic acid. J. Agric. Chem. Soc. Japan., 35: 219-223. 


\section{Nour El-Din et al.}

Van Loon, L.C. (2007). Plant responses to plant growth-promoting rhizobacteria. Eur. J. Plant Pathol. 119, 243-254.

Voss, H. D. (1992). Relating colormeter measurements of plant color to the Royal Horticultural Society color chart. Hort. Science, 27 (12):12561260.

Yicheng Tu (2000). Endogenous gibrillins in developing apple seeds in relation to alternate bearing. Ms. C Thesis, Faculty of Purdue University.

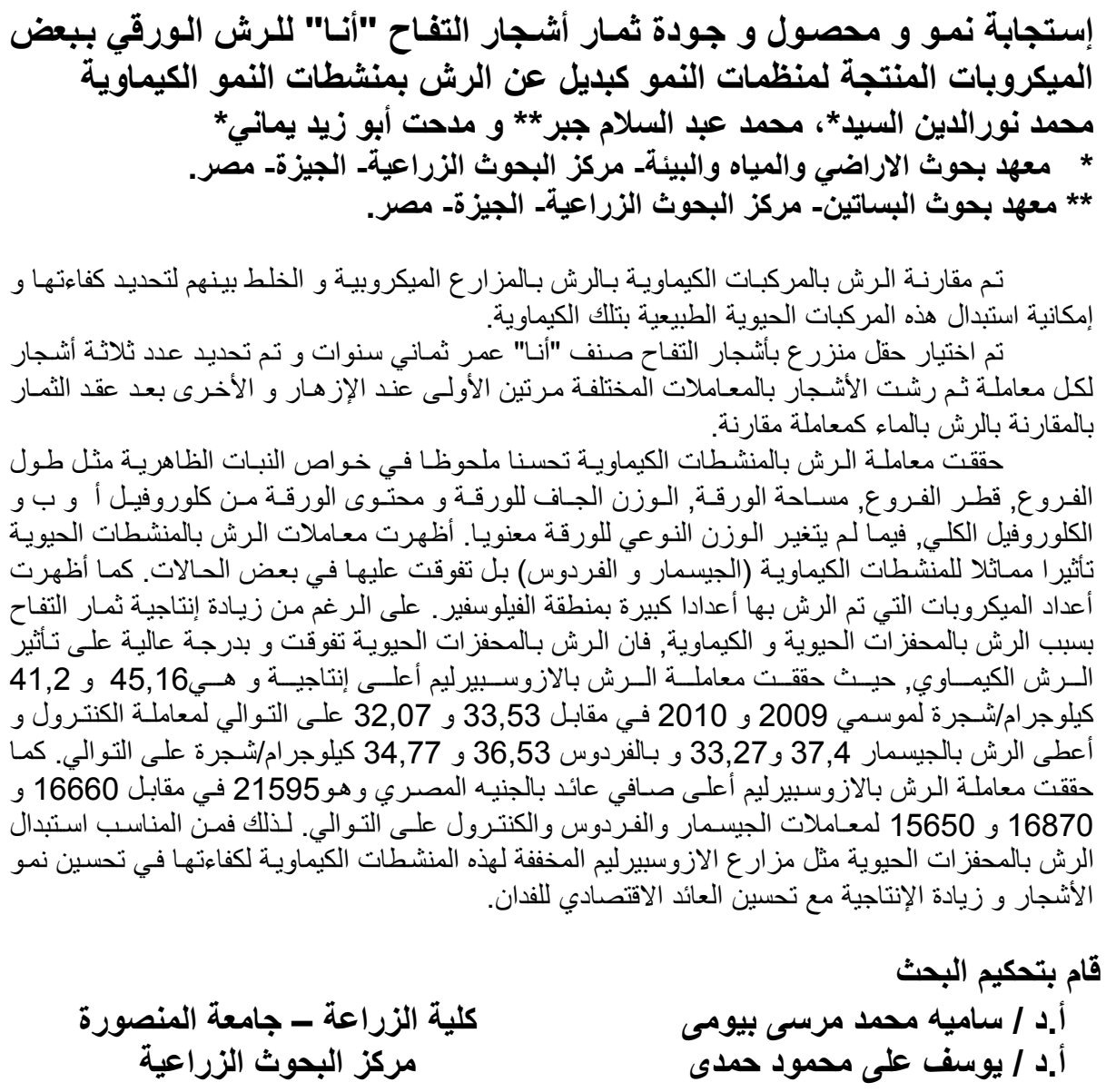

كلية الزراعة - جامعة المنصورة

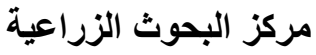

